Les ANNALES Les Annales de droit

DE DROIT

13 | 2019

Varia

\title{
Le contrôle juridictionnel des mesures de police en droit administratif camerounais
}

How jurisdictions control police decisions in administrative law in Cameroon

Éric-Adol T. Gatsi

\section{(2) OpenEdition}

Journals

Édition électronique

URL : http://journals.openedition.org/add/1529

DOI : $10.4000 /$ add. 1529

ISSN : 2606-1988

Éditeur

Presses universitaires de Rouen et du Havre

Édition imprimée

Date de publication : 1 mai 2019

Pagination : $89-120$

ISBN : 979-10-240-1293-3

ISSN : 1955-0855

Référence électronique

Éric-Adol T. Gatsi, « Le contrôle juridictionnel des mesures de police en droit administratif camerounais », Les Annales de droit [En ligne], 13 | 2019, mis en ligne le 09 décembre 2019, consulté le 24 janvier 2021. URL : http://journals.openedition.org/add/1529; DOI : https://doi.org/10.4000/add. 1529

Ce document a été généré automatiquement le 24 janvier 2021.

Presses universitaires de Rouen et du Havre 


\title{
Le contrôle juridictionnel des mesures de police en droit administratif camerounais
}

\author{
How jurisdictions control police decisions in administrative law in Cameroon
}

\author{
Éric-Adol T. Gatsi
}

1 Définir l'ordre public présente des difficultés en raison de la diversité de ses utilisations, mais surtout de la pluridisciplinarité qui le caractérise. Toutes les branches $\mathrm{du}$ droit et toutes les disciplines du droit se l'approprient en lui donnant un contenu et une signification différente ${ }^{1}$. Renvoyant, en droit privé, à l'ensemble des règles d'une importance particulière sur lesquelles on ne peut transiger ${ }^{2}$, l'ordre public se décline quasiment exclusivement en terme d'exception, de réserve en droit communautaire dans la mesure où son évocation conduit à ce que les États membres d'une communauté s'opposent, "sous certaines conditions, à l'exercice intégral des libertés reconnues ${ }^{3}$ " par les textes communautaires ${ }^{4}$. Défenseur «des valeurs fondamentales de l'État du for $^{5} »$ en droit international privé 6 ; il désigne, en droit international public, les normes supérieures de droit international, insusceptibles de dérogation et considérées comme le « "soubassement" juridique de la société internationale ${ }^{7}$ ». En matière procédurale enfin, l'ordre public joue le rôle de "police du système juridique ${ }^{8}$ " en ceci que son évocation renvoie à des règles tellement importantes que leur application devient obligatoire, le juge pouvant même les soulever d'office.

2 C'est l'ordre public en droit administratif qui constitue l'objet de cette étude, à savoir celui qui s'exprime à travers la police administrative dans son acception matérielle qu'est « l'activité consistant à prévenir les troubles à l'ordre public et à maintenir celui$\mathrm{ci}^{9}{ }$. Il est important de relever la progressivité dans la formation du contenu de l'ordre public en France dont la conception camerounaise s'inspire. Partant d'un ordre public dans la rue, visible et extérieur ${ }^{10}$ renvoyant à la trilogie sécurité, tranquillité et salubrité publiques, l'ordre public s'est enrichi, avec les arrêts Société Les films Lutétia et ceux dits du "lancer du nain ${ }^{11}$ ", des considérations de valeur qui lui donne une dimension axiologique désormais insoupçonnable. Au Cameroun, la situation est 
quelque peu différente, tant en ce qui concerne l'ordre public matériel que relativement à l'ordre public immatériel.

D'une part, sur l'ordre public matériel, les textes parlent tantôt uniquement « d'ordre public », tantôt d'« ordre public, de sécurité publique et de tranquillité publique ${ }^{12}$ ». L'utilisation non uniforme des référents à l'ordre public amène à s'interroger sur son réel contenu. L'ordre public doit-il être compris séparément de la sécurité et la tranquillité publiques, ou celles-ci doivent-elles être considérées comme les composantes de celui-là ? Qu'en est-il de la salubrité, la troisième composante de l'ordre public matériel en France, mais que les textes camerounais passent sous silence ? Son absence signifie-t-elle son exclusion du contenu de l'ordre public? Si cette rédaction des textes est à déplorer, il convient surtout de la considérer comme une invitation du juge, interprète authentique de la loi, à définir avec précision le contenu de l'ordre public. À ce titre, le juge $a$, dans l'espèce Nguessie $^{13}$ reconnu de manière expresse que la salubrité publique était bien une composante de l'ordre public.

D'autre part, le débat sur l'existence d'un ordre public moral qui a longtemps existé en France est écarté dès l'origine par la Constitution camerounaise, qui associe à l'ordre public la notion de "bonnes mœurs ${ }^{14}$ », reconnaissant ainsi l'existence d'un ordre public moral. Le fondement de l'ordre public moral est donc, au Cameroun, textuel, alors qu'il est exclusivement prétorien en France ${ }^{15}$.

5 L'une des questions centrales liées à l'étude de la notion d'ordre public en droit administratif est relative aux rapports qu'il entretient avec les libertés publiques. Si l'on peut affirmer, comme Bernard Stirn, qu'ordre public et libertés publiques «se comprennent mieux ensemble que séparément ${ }^{16} "$, l'agencement de leurs rapports révèle la complexité de la question qui se décline en deux points essentiels : la fonction de l'ordre public par rapport aux libertés et la hiérarchie entre l'ordre public et les libertés.

6 Sur le premier point, à la question de savoir si l'ordre public constitue une menace ou une garantie pour les libertés, deux écoles de pensée s'affrontent. Pour certains, parce qu'il s'emploie toujours en termes de restriction, de limitation, d'interdiction, l'ordre public est, par nature, une menace aux libertés publiques ${ }^{17}$. Pour d'autres, parce qu'il ne peut y avoir de libertés dans le désordre, l'ordre public est une condition pour un meilleur épanouissement des libertés, ce qui a d'ailleurs été confirmé par le Conseil constitutionnel français qui, dans sa décision sur la Loi relative à l'État d'urgence en Nouvelle-Calédonie, a affirmé la nécessité impérieuse de l'ordre public «sans lequel l'exercice des libertés ne saurait être assuré18 ». Pourtant, si ces deux thèses antinomiques s'appuient sur des arguments plus ou moins pertinents, il reste qu'il est nécessaire de dépasser la confrontation entre ordre public et libertés publiques qui ne rend pas réellement compte de la réalité, en ce sens qu'elle oppose deux notions qui ne sont pas opposées. Ce qui amène à la thèse de la consubstantialité soutenue par Étienne Picard, pour qui l'ordre public n'est pas l'antithèse des libertés, mais une «norme générale et abstraite qui habite l'idée de droit au même titre que le primat de la liberté », car «la liberté juridique inclut nécessairement des exigences d'ordre public ${ }^{19}$ ».

7 Sur la question de la primauté entre l'ordre public et les libertés, la thèse de la nécessité de principe s'oppose à celle de la nécessité pratique ${ }^{20}$. La première, qui présuppose le primat des libertés sur l'ordre public, présente la liberté comme une valeur pré-étatique, naturelle et consubstantielle à tout être humain, inviolable et 
sacrée. Ce fondement philosophique de la primauté de la liberté sur l'ordre public est complété par un autre fondement théorique découlant des connotations de l'adoption des mesures d'ordre public : « la référence à l'ordre public suppose toujours l'existence d'un état normal "momentanément" suspendu pour des raisons pratiques ${ }^{21}$. " Ainsi, en se présentant comme un droit exceptionnel susceptible de justifier la restriction aux libertés, l'ordre public confirme ipso facto que celles-ci constituent la règle : l'exception (de l'ordre public) confirme la règle (des libertés).

Cette thèse de la primauté des libertés sur l'ordre public trouve difficilement une justification en droit, car elle se fonde davantage sur des considérations idéologiques, philosophiques qui font ainsi de la primauté des libertés sur l'ordre public un principe plus métajuridique que véritablement juridique. C'est pourquoi certains auteurs lui préfèrent la thèse de la nécessité pratique de l'ordre public, qui fonde la primauté de l'ordre public sur son but suprême qu'est l'intérêt supérieur de l'État, l'équilibre d'un système, la survie de l'État, la paix sociale. Comme le dit Thomas Dumortier, «le présupposé d'un réalisme conceptuel attaché aux justifications de l'ordre public appelle à $\mathrm{y}$ voir une norme fondamentale, susceptible de fonder des dérogations " variables selon la gravité de la situation. Philippe Terneyre voit en l'ordre public le fondement d'une obligation de "s'affranchir des règles constitutionnelles pour assurer la continuité de la vie nationale », reconnaissant ainsi à l'ordre public une nécessité de nature « supraconstitutionnelle ${ }^{22}$ ».

9 Sur cette question, les deux thèses en présence n'emportent pas entièrement satisfaction. La détermination d'une hiérarchie entre l'ordre public et les libertés publiques est d'autant plus complexe qu'il s'agit d'établir un ordre de priorité entre deux notions également protégées par la Constitution camerounaise ${ }^{23}$. À ce titre, l'ordre de priorité entre elles doit se faire de manière prudente, en émettant des hypothèses. $\mathrm{Si}$ l'on doit reconnaître, malgré leur posture juridique égale, à l'une de ces notions une valeur intrinsèque supérieure à l'autre, il sera difficile de ne pas la reconnaître au profit de l'ordre public dès lors qu'il renvoie au maintien d'un équilibre social. En revanche, si l'on part du postulat qu'il ne peut y avoir de hiérarchie présupposée entre ces deux notions constitutionnellement consacrées et protégées, il convient de reconnaître que l'ordre des priorités ne peut être déterminé que par le juge, selon le cas. Cette hypothèse plus concrète est plus satisfaisante, car la détermination d'un ordre de priorité entre l'ordre public et les libertés ne peut se faire comme un principe figé, gravé dans le marbre, qui doit s'appliquer en toutes circonstances. Elle doit, au contraire, s'arrimer avec la situation de fait. Le tandem ordre public-libertés publiques obéit à des logiques différentes selon la période considérée, voire selon les priorités identifiées.

10 C'est tout l'intérêt de cette étude, qui part du constat de l'évolution de la relation entre l'ordre public et les libertés publiques au Cameroun sur le plan textuel. On est passé d'un agencement profitant exclusivement à l'ordre public dès le lendemain des indépendances, dans un contexte dominé par la construction de l'unité nationale à une dynamique de plus en plus libérale depuis le début des années 1990, à la faveur d'une libéralisation de la vie politique ${ }^{24}$. Les lois libérales adoptées dès les années 1990 ont marqué le choix du changement de cap, du tout sécuritaire vers une direction plus favorable pour les libertés. En conséquence, les pouvoirs de police jadis étendus ont été considérablement réduits, en même temps que certaines mesures coercitives ont été supprimées. Le législateur a ainsi aménagé la conciliation entre l'ordre public et les 
libertés, conciliation dont le contrôle revient au juge. C'est en effet lui qui, en définitive, donne une signification concrète à cette conciliation et en fixe la portée et les limites.

11 Il sera donc question, dans cet article, de comprendre comment s'agence le contrôle des actes de police au Cameroun. Tel qu'il est prévu et mis en œuvre, le contrôle effectué sur les actes de police est-il de nature à instituer une conciliation satisfaisante entre l'ordre public et les libertés publiques? La réponse à ce questionnement commande que l'on passe en revue à la fois les dispositions législatives régissant ce contrôle et la politique jurisprudentielle du juge dans la mise en œuvre de celui-ci. À ce titre, l'hypothèse retenue est celle-ci: malgré le mouvement de libéralisation de la vie politique entamé depuis le début des années 1990, la conciliation entre l'ordre public et les libertés publiques révèle un déséquilibre en faveur de l'ordre public, qui se traduit par une mitigation de la conciliation sur le plan textuel (1) et une minoration de celle-ci sur le plan matériel par les choix jurisprudentiels (2).

\section{Une conciliation mitigée sur le plan textuel}

12 L'idée est de passer au crible l'agencement des mécanismes de conciliation, tels qu'ils sont prévus par les textes en vigueur. À ce titre, le caractère mitigé de cette conciliation découle du fait que les mécanismes de conciliation ne sont pas suffisamment à même de protéger les libertés contre les pouvoirs de police de l'administration, car malgré une libéralisation de la procédure en matière d'urgence (1.2), on note une rédaction des textes sur la juridiction compétente (1.1) susceptible de produire des effets néfastes.

\subsection{L'imbroglio juridique autour de la juridiction compétente}

De manière générale, si l'on part de l'hypothèse selon laquelle le juge administratif est le juge de l'administration, la problématique de la détermination de la juridiction compétente en matière d'actes de police peut sembler superflue et sans intérêt. La situation est, en pratique, plus complexe dans la mesure où le législateur a toujours institué un système mixte alliant la clause générale de compétence à l'énumération ${ }^{25}$, ce qui amène à relativiser le postulat du juge administratif, juge naturel de l'administration ${ }^{26}$. L'agencement des compétences de l'un et de l'autre peut déboucher sur une confusion de leurs domaines d'action respectifs, comme cela a été le cas en matière de contentieux relatif aux réunions et manifestations (1.1.1) et de celui lié à la communication sociale (1.1.2).

\subsubsection{L'imprécise compétence en matière de contentieux relatif aux réunions et manifestations publiques}

14 La loi portant régime des réunions et manifestations publiques précitée fixe le régime du contentieux relatif à ces matières. À ce sujet, il faut clairement distinguer la situation liée aux réunions publiques de celle liée aux manifestations publiques. Pour ces dernières, l'article 8(3) et (4) confie le contentieux de l'interdiction au président du Tribunal de grande instance territorialement compétent qui siège sous huitaine et dont l'ordonnance est susceptible de recours selon les conditions de droit commun. Quant aux réunions publiques, aucune disposition n'est prévue pour son contentieux. Cette omission n'est pas un oubli, puisque le législateur n'a pas prévu d'hypothèse 
d'interdiction d'une réunion publique qui ne peut être suspendue ou interrompue qu'à la seule initiative du bureau organisateur. La difficulté consiste à déterminer si cette omission est synonyme de l'exclusion de toute intervention judiciaire en matière de réunion publique.

La question s'est posée de manière indirecte dans l'affaire Moubarak Ibrahim Mbombo ${ }^{27}$, du nom de ce ministre du culte dont la réunion légalement déclarée, moyennant récépissé, avait plus tard été interdite et le récépissé annulé par un arrêté du souspréfet. Saisi aux fins d'annulation dudit arrêté, le juge administratif, après avoir rappelé les dispositions de l'article 8 précité, se déclara incompétent en considérant que «c'est le juge de droit commun qui est compétent pour statuer sur les contestations résultant des décisions de l'autorité administrative en ce qui concerne les réunions ou manifestations publiques ». Cette décision dénote une confusion totale des régimes des réunions et manifestations publiques, tant du point de vue de la compétence du juge que de celui des pouvoirs de l'autorité de police ${ }^{28}$. Comme on la vu, le législateur n'a pas prévu de contentieux en matière de réunions publiques puisqu'il n'a pas prévu d'intervention de l'administration. Le mutisme du législateur est, à ce sujet, critiquable puisqu'il présuppose l'inexistence des litiges en cette matière, ce que l'affaire Ibrahim Moubarak Mbombo a démenti.

Qui est donc compétent en cas de litige lié aux réunions publiques? Le mutisme de la loi peut-il conduire à un raisonnement par analogie : la compétence du juge judiciaire explicitement reconnue en matière de contentieux des manifestations publiques peutelle être étendue en matière de contentieux des réunions publiques? C'est cette solution qui a été adoptée par le juge dans sa décision, celui-ci ayant estimé que les dispositions de l'article 8(3) et (4), qui confient le contentieux de l'interdiction d'une manifestation publique au juge judiciaire, sont applicables par extension en matière de réunion publique. Pourtant, elle est loin d'être satisfaisante, puisqu'elle fait dire au législateur ce qu'il n'a manifestement pas voulu dire. C'est dire que le raisonnement par analogie est ici peu indiqué. L'on pourrait plutôt penser que la compétence du juge judiciaire étant, en droit administratif, dérogatoire, on ne peut l'étendre à des matières non expressément prévues par le législateur. Ainsi, l'absence de mention explicite de la compétence du juge judiciaire en matière de contentieux des réunions publiques doit être interprétée comme une attribution tacite au «juge ordinaire des actes administratifs ${ }^{29} »$ qu'est le juge administratif $^{30}$. C'est dire que le juge de l'espèce aurait dû se déclarer compétent, sa décision d'incompétence s'analysant comme un déni de justice très préjudiciable aux justiciables.

\subsubsection{L'illisible compétence en matière de contentieux de la liberté de presse}

17 La rédaction imprécise et floue des dispositions relatives à la juridiction compétente en matière de police de la presse rend illisibles ces règles de compétence. Tout part de l'article 14 de la première mouture de la loi relative à la communication sociale au Cameroun précitée qui confiait le contentieux de la censure, de la saisine et de l'interdiction des organes de presse au « juge compétent » devant statuer dans un délai d'un mois. La difficulté consistait à déterminer à quel juge le législateur faisait allusion par cette disposition plus que sibylline et propre à décourager les justiciables ${ }^{31}$. La réponse à cette difficulté a évolué au gré de l'évolution législative.

En 1991, c'est-à-dire sous la première mouture de la loi de 1990 sur la communication sociale, le juge administratif a eu à se prononcer indirectement sur la question dans 
l'affaire Le Messager ${ }^{32}$. À la suite de la censure de certains articles du numéro 218 de son journal pour menace à l'ordre public, le directeur de publication du Messager saisit le juge des référés administratif à l'effet de lever cette mesure de censure et permettre la diffusion des informations qu'il considère, à juste titre, comme des «denrées rapidement périssables». Le juge administratif se déclarera incompétent sous le prétexte que l'article 14 précité ne prévoyait pas de procédure de référé en matière de censure des journaux. Si la décision d'incompétence est correcte, on ne peut en dire de même du motif sur lequel le juge l'adosse, à savoir l'absence de prévision de la procédure de référé par la loi sur la communication sociale. Cette motivation est d'autant moins pertinente que l'article 122 de la loi n ${ }^{\circ}$ 75/17 du 8 décembre 1975 fixant procédure devant la Cour suprême statuant en matière administrative prévoyait bel et bien cette procédure "dans tous les cas ", excepté ceux qui intéressent l'ordre public. C'est dire que c'est davantage cette exception liée à l'ordre public - par ailleurs soulevée par le représentant de l'État- qui aurait dû motiver la décision d'incompétence du juge en l'espèce. Ainsi, le contentieux de l'interdiction d'un organe de presse ne pouvait donc se faire que sur le fond ${ }^{33}$, comme toutes les autres matières intéressant l'ordre public.

Peut-on déduire de cette décision une certaine reconnaissance par le juge administratif de sa compétence en matière principale ? Cette conclusion qui a été tirée par certains auteurs ${ }^{34}$ semble bien envisageable. En d'autres termes, en déclinant sa compétence en matière d'urgence, le juge administratif de céans reconnaissait implicitement qu'il était bien le « juge compétent » de l'article 14 précité puisque ces griefs rentraient bien dans le cadre de sa compétence telle qu'elle est fixée par l'article 9 de l'ordonnance précitée. Cependant, l'exclusion de la procédure de référé par le juge administratif avait pour conséquence d'instituer une immunité de fait des actes de police en matière de presse. Ainsi exclu en matière d'urgence, le recours contre la censure d'un organe de presse ne pouvait se faire devant lui qu'au principal. Or, le même article 14(5) prévoyait un délai de jugement d'un mois à compter de la saisine en cas de censure, de saisie ou d'interdiction. S'agissant de quotidiens ou d'hebdomadaires traitant d'informations urgentes et périssables ${ }^{35}$, ce délai rendait une telle procédure inutile et inefficace. Dans ces conditions, la seule voie de contestation possible d'une mesure de censure, d'interdiction ou de saisie d'un organe de presse était le recours devant le juge judiciaire, statuant en urgence.

20 En 1996, à la faveur de la révision de la loi sur la communication sociale précitée, l'expression vague du «juge compétent » est maintenue, mais le législateur précise que ce juge devra être saisi « en référé d'heure en heure ou suivant les dispositions légales analogues dans les provinces du Nord-Ouest et du Sud-Ouest ${ }^{36}$ ». La problématique de la détermination du juge compétent demeure et trois solutions sont envisageables. La première consiste à entendre par " juge compétent » le juge administratif, juge naturel des actes administratifs ${ }^{37}$. L'on pourrait ainsi penser qu'en rectifiant le tir par l'institution d'un délai de jugement parfaitement compatible avec l'urgence qui caractérise le contentieux des organes de presse, le législateur a permis un recours utile devant le juge administratif. Dans cette logique, l'intervention du juge judiciaire imaginée plus haut comme palliatif à l'ineffectivité du recours devant le juge administratif serait désormais sans fondement.

Cette solution qui aurait l'avantage de maintenir le contentieux administratif devant le juge administratif peut être contredite par une autre lecture des dispositions en 
question. En effet, la deuxième solution procède d'une autre logique : l'évocation, d'une part, de la procédure de référé d'heure en heure inconnue du droit administratif camerounais, et d'autre part des procédures légales analogues en vigueur dans la partie anglophone du pays, où les règles de la procédure administrative contentieuse sont alors inconnues et inappliquées ${ }^{38}$, pourrait amener à penser que le législateur a vraisemblablement voulu confier ce contentieux au juge judiciaire. Ainsi, le juge compétent de l'article 17 serait le juge judiciaire. Cette solution a été adoptée par le juge de l'affaire Mutations intervenue en $1997^{39}$. Dans cette espèce, après la décision de saisie d'un de ses numéros par le gouverneur de la province du Centre, le directeur de publication de ce journal saisit le juge judiciaire des référés, en l'occurrence le président du Tribunal de première instance, qui reconnut sa compétence sur la base de l'article 17(2) précité, en dépit de l'argumentaire du représentant de l'État qui l'invitait à décliner sa compétence au profit du juge administratif. Cette compétence sera confirmée par le juge d'appel du Centre, saisi par l'État ${ }^{40}$. Cette solution a été très critiquée par certains auteurs qui décrient l'empiétement par le juge judiciaire du champ de compétence du juge administratif ${ }^{41}$.

Quoi que s'appuyant sur des arguments plus ou moins pertinents, ces deux solutions n'emportent pas totalement satisfaction. D'un point de vue téléologique, il paraît clair que le législateur a voulu instituer une procédure qui tient compte des réalités du système bi-juridique et donc bi-juridictionnel camerounais. À ce titre, les deux fragments de cette disposition rendent compte des deux sous-systèmes juridiques latino-romains dans la partie francophone et anglo-saxonne dans la partie anglophone du pays : le référé d'heure en heure est appliqué dans la partie francophone par le juge compétent en matière administrative qui est le juge administratif; les procédures analogues en vigueur dans les provinces du Nord-Ouest et du Sud-Ouest sont applicables dans la partie anglophone ${ }^{42}$ qui, fidèle à la tradition anglo-saxonne de la Common law, ne connaît pratiquement pas, alors, la distinction droit privé-droit public. Il s'agissait donc, dans cette partie du pays, du juge judiciaire ${ }^{43}$.

Il est permis de croire que la mise en place effective des tribunaux administratifs dans les chefs-lieux de régions, par décrets $\mathrm{n}^{\circ}$ 2012/119 et 194 des 15 mars et 18 avril 2012, portant, respectivement ouverture des tribunaux administratifs et nomination des magistrats du siège dans lesdits tribunaux ${ }^{44}$, a changé la donne. En effet, ces décrets mettent en application l'article 40 du texte constitutionnel de 1996 qui créait des «juridictions inférieures en matière de contentieux administratif ». Le processus de mise en place de ces juridictions inférieures avait déjà commencé avec l'adoption de la loi $n^{0}$ 2006/022 du 29 décembre 2006 fixant l'organisation et le fonctionnement des tribunaux administratifs qui innovait en reconnaissant explicitement au profit du juge administratif le contentieux des litiges intéressant les opérations de maintien de l'ordre ${ }^{45}$. Il y'a donc un conflit entre la loi de 1990, modifiée en 1996, et la loi de 2006 précitée. La première institue une distinction procédurale sur la communication sociale en matière de recours contre les mesures de police prises à l'encontre d'un organe de presse, selon que l'on se trouve dans le Cameroun anglophone ou dans le Cameroun francophone. La seconde, tout en unifiant la procédure administrative contentieuse, pose comme une clause générale de compétence au profit du juge administratif en matière de contentieux lié à l'ordre public.

Deux solutions à ce conflit de lois sont envisageables. La règle de la lex specialis conduirait à affirmer que la loi de 2006, étant une loi générale qui traite du contentieux 
de la police administrative dans son ensemble, ne peut s'appliquer en présence d'une loi spéciale qui traite du contentieux d'une police administrative spéciale, en l'occurrence celle liée aux organes de presse ${ }^{46}$. Vue sous cet angle, la loi de 1990 resterait applicable et la distinction procédurale serait maintenue, relativement à la juridiction compétente en matière de contentieux des mesures de police contre un organe de presse. Cette solution, qui a l'inconvénient d'instituer une procédure à deux temps, est par ailleurs contraire à la volonté du constituant de 1996 qui a voulu harmoniser la procédure administrative sur toute l'étendue du territoire national ${ }^{47}$. C'est pourquoi la règle de la lex posterior, qui porte sur la chronologie dans le règlement des conflits de lois ${ }^{48}$, semble plus adéquate pour le règlement de ce conflit. Si la distinction faite par le législateur dans la loi sur la communication sociale se fondait sur les différences procédurales dans la partie francophone et dans la partie anglophone du pays, l'uniformisation de la procédure administrative contentieuse sur l'ensemble du territoire national, avec l'institution de ces tribunaux administratifs déconcentrés, rend cette distinction désuète. En conséquence, en mettant un terme à ce doublon procédural, la mise en place effective des tribunaux administratifs a institué un régime unique de compétence en matière d'actes de police en général, et spécialement ceux pris en matière de liberté de la presse, en faveur du juge administratif.

\subsection{La libéralisation progressive de la procédure contentieuse d'urgence en matière de mesures de police}

La libéralisation de la procédure contentieuse est une constante dans l'ensemble du contentieux administratif camerounais. On ne s'attardera donc qu'aux cas ayant une incidence sur l'aménagement des relations ordre public-libertés. Ainsi, parce qu'elles trouvent, en matière de mesures de police leur expression la plus parfaite, les procédures d'urgence retiennent notre attention ${ }^{49}$ et à ce titre, on note une immunité provisoire des mesures de police relative aux procédures d'urgence accessoires (1.2.1) et l'institution des garanties procédurales en matière d'urgence spéciale (1.2.2).

\subsubsection{L'immunité provisoire des mesures de police relativement aux procédures d'urgence accessoire}

Les procédures d'urgence accessoires sont celles engagées parallèlement à l'instance principale et qui ont pour but d'empêcher que l'exécution des décisions administratives pose des dommages inconsidérés. Il s'agit du sursis à exécution et du référé administratif. Alors que le premier, restriction au principe de l'effet non suspensif du recours contre la décision administrative, tend à en suspendre l'exécution en attendant que le juge statue sur le fond ${ }^{50}$, le second vise la protection des intérêts des particuliers que l'exécution des mesures édictées pourrait mettre en péril, à travers des mesures conservatoires $^{51}$.

Si ces procédures ne sont pas propres aux cas des mesures édictées dans le cadre de l'ordre public, il demeure que le contentieux de ces mesures de police leur donne une particularité, notamment en ce qui concerne l'immunité provisoire des mesures de police et les conditions de déroulement de l'instance.

D'abord, sur l'immunité des mesures de polices, les articles 16 et 122 de la loi n ${ }^{\circ} 75 / 17$ du 8 décembre 1975 fixant la procédure devant la Cour suprême statuant en matière 
administrative ne permettaient le sursis à exécution et le référé administratif de décisions administratives que dans les cas qui n'intéressent pas l'ordre public. La loi de 2006 précitée fixant l'organisation et fonctionnement des tribunaux administratifs a quelque peu tempéré cette interdiction, en levant cette restriction pour le référé, ne la maintenant que pour le sursis à exécution ${ }^{52}$. Ainsi, hormis le cas du référé administratif depuis 2006, les mesures de police bénéficient d'une immunité juridictionnelle provisoire complète, en ce sens qu'ils ne peuvent être remis en cause que devant le juge du principal. Cette présomption de légalité accordée aux mesures de police s'explique sans doute par le but de la police administrative qui est la protection de l'ordre public, lequel peut être menacé pendant le procès au principal. C'est la raison pour laquelle cette immunité a été levée concernant le référé administratif, puisque contrairement au sursis à exécution qui empêche à la mesure d'être exécutée, il ne fait pas véritablement obstacle à ce que la mesure édictée soit mise en application ${ }^{53}$. Le maintien de l'exclusion du sursis à exécution des mesures de police pourtant "particulièrement utile à la défense des droits et libertés ${ }^{54}$ " et qui traduit la sacralisation des mesures d'ordre public est considéré comme un obstacle à une meilleure conciliation par le juge de l'ordre public et des libertés.

On peut se demander si le juge est lié par la référence à l'ordre public de l'autorité administrative, ou alors s'il dispose en l'espèce d'un pouvoir d'appréciation. La formule devenue rituelle employée dans les ordonnances de sursis à exécution par le juge ${ }^{55}$ peut laisser penser à un droit d'appréciation de sa part. La réalité a évolué au gré de l'évolution législative et même constitutionnelle. La loi de 1975 précitée conditionnait la décision accordant le sursis à exécution et le référé administratif à l'avis conforme du Ministère public. Ainsi, soulevée par l'administration, l'évocation du lien avec l'ordre public était donc de droit et liait le juge si elle était acquiescée par le Ministère public. Comme l'a relevé à juste titre Célestin Keutcha Tchapnga, « la simple référence à une menace à l'ordre public est une motivation suffisante pour rendre pratiquement impossible l'obtention d'un sursis à exécution ${ }^{56} \%$. La révision constitutionnelle du 18 janvier, en érigeant l'autorité judiciaire en pouvoir judiciaire, a amené le juge à s'émanciper de la tutelle du Ministère public. Dans certaines décisions en effet, le juge démontre sa liberté vis-à-vis de l'avis du ministère public: "vue la disposition du titre 5 de la loi $n^{\circ}$ 96/6 du 18 janvier 1996 portant révision de la Constitution ayant érigé l'autorité judiciaire en pouvoir judiciaire, vue l'ensemble des dispositions des articles 37 et 68 de ladite Constitution qui modifie substantiellement la portée des dispositions de l'article 16 de la loi $\mathrm{n}^{\circ} 75 / 17$ du 8 décembre 1975 fixant la procédure devant la Cour suprême statuant en matière administrative ${ }^{57} . .$. » Cette émancipation prétorienne sera avalisée par le législateur qui supprimera purement et simplement cette condition liée à l'avis conforme du Ministère public dans la loi précitée de 2006, portant organisation et fonctionnement des Tribunaux administratifs.

\subsubsection{L'institution de procédures d'urgence spéciale en matière d'ordre public}

Le contentieux administratif spécial est constitué d'un certain nombre de matières, à propos desquelles le législateur a institué une procédure dérogatoire du droit commun de la procédure administrative dans le sens d'une libéralisation plus poussée. À l'observation, cette tendance n'a cours que depuis 1990, ce qui témoigne d'une prise de conscience de la nécessité pour le législateur d'une meilleure prise en compte des libertés dans la procédure contentieuse. Relativement à l'ordre public, quatre domaines 
de libertés sont soumis à ce régime dérogatoire et allégé : la liberté de la presse, la liberté d'association et des organisations non gouvernementales, la liberté partisane et la liberté des réunions et manifestations publiques, qui s'illustrent depuis 1990 par deux dérogations notoires, à savoir l'absence de l'exigence du recours gracieux préalable et l'institution d'une célérité dans l'office du juge.

31 La levée de l'exigence du recours gracieux préalable, qui est une condition substantielle de recevabilité de la requête dans le contentieux administratif ordinaire ${ }^{58}$, est une grande avancée pour les contentieux des libertés liées à l'ordre public, en ce sens qu'elle permet un accès plus facile et rapide au juge ${ }^{59}$. On sait que le recours gracieux préalable est effectué dans les trois mois suivant la publication ou la notification de la mesure attaquée, l'autorité destinataire ${ }^{60}$ ayant jusqu'à trois mois pour rendre sa décision ${ }^{61}$. Au demeurant, le juge peut être saisi jusqu'à six mois après l'adoption de la mesure attaquée, ce qui constitue un délai extrêmement long dans des domaines où la célérité est une exigence capitale. Le législateur affranchit donc les justiciables de l'obligation d'effectuer le recours gracieux préalable dans le contentieux relatif à ces différentes matières, ce que le juge respecte plutôt scrupuleusement ${ }^{62}$.

La célérité qui constitue le maitre-mot de cette entreprise de libéralisation par le législateur est également visible dans l'action même du juge. D'abord, son office est désormais enserré dans des délais très contraignants, qui prennent en considération la nécessité impérieuse de rendre une décision le plus tôt possible, en fonction du cas considéré : 2 heures ou 1 jour pour le contentieux de la saisie ou de l'interdiction d'un organe de presse, selon qu'il s'agit d'un quotidien ou d'un périodique ; 8 jours pour le contentieux de l'interdiction d'une réunion publique ; 10 jours pour le contentieux de la suspension ou de la dissolution d'une association; 30 jours pour le contentieux du refus de légalisation d'un parti politique, ainsi que pour le contentieux de dissolution d'une organisation non gouvernementale ${ }^{63}$. Ces prescriptions sont d'autant plus importantes et notables qu'elles sont dérogatoires de la procédure administrative contentieuse ordinaire qui, en général, ne fixe aucun délai au juge pour vider sa saisine, ce qui rend les délais de jugement des affaires devant le juge administratif extrêmement longs ${ }^{64}$, et les décisions souvent inutiles ${ }^{65}$.

Ensuite, contrairement au principe de la collégialité qui caractérise la justice administrative en temps ordinaires, le législateur institue l'exception du juge unique, comme dans le cas des procédures d'urgence accessoires. La véritable prouesse du législateur, à travers l'institution de ces procédures d'urgence spéciales, est d'avoir lié le principal et l'accessoire puisqu'il institue une procédure d'urgence pour statuer sur le fond, à savoir que le juge saisi selon une procédure d'urgence ne se contente plus de prendre une mesure conservatoire, mais tranche le litige de manière complète. Cette dynamique, qui va dans le sens d'une meilleure justice administrative en matière de mesures de police, va être rompue par les choix jurisprudentiels du juge.

\section{Une conciliation minorée sur le plan jurisprudentiel}

"Le contrôle du juge sur la définition de l'ordre public est un enjeu fondamental ${ }^{66}$ " puisque c'est lui qui, en définitive, donne un sens concret à la conciliation de l'ordre public et des libertés prescrite par le législateur. Au Cameroun, malgré l'amorce d'une libéralisation globale de la vie sociopolitique, et sans aller jusqu'à affirmer qu'il n'exerce qu' « un contrôle purement formel ${ }^{67}$ ", le juge de la légalité ${ }^{68}$ des mesures de 
police effectue un contrôle minimum. Ce choix est fortement dicté par la nature même de l'ordre public, puisque de l'avis de Bertrand Seiller, le contrôle minimum « est mis en œuvre par le juge en raison de l'imprécision des catégories abstraites, de la technicité particulière des appréciations à porter ou du caractère sensible du domaine de l'action administrative ${ }^{69} \%$. Si, en général, le contrôle de la légalité interne de l'acte ne pose pas de problème particulier ${ }^{70}$, il en va autrement de sa légalité externe où le juge met en œuvre un contrôle des faits (2.1) et du contenu des mesures (2.2) qui laisse plus d'un insatisfait.

\subsection{L'insuffisant contrôle des faits justifiant les mesures de police}

Le contrôle par le juge des faits traduit l'idée selon laquelle son contrôle ne se confine pas seulement au contenu des mesures édictées, puisqu'il lui permet d'apprécier les " circonstances de fait dont l'existence conditionne la validité ou la légalité de l'acte ${ }^{71}$ ». C'est à travers le contrôle de la qualification juridique des faits et celui de leur matérialité que le juge fait son immixtion dans le contrôle des faits justifiant les mesures de police ${ }^{72}$.

\subsubsection{Le contrôle permissif de la qualification juridique des faits}

Élément du contrôle normal ${ }^{73}$, le contrôle de la qualification juridique des faits amène le juge à confronter les faits, réalité concrète, au droit, entité abstraite. En contrôlant la qualification juridique des faits, le juge administratif s'assure que la situation de fait en cause rentre bien dans la catégorie juridique dans laquelle l'administration veut la mettre $^{74}$.

Admis en France depuis l'arrêt Gomel $^{75}$, le contrôle de la qualification juridique des faits revêt une importance capitale dans le contrôle de l'action gouvernementale, en raison «de la fréquente nécessité de contrôler la qualification juridique des faits et de la fréquence des erreurs commises par les autorités administrative ${ }^{76} »$. Devant le juge administratif camerounais et en matière de police administrative, le contrôle de la qualification juridique des faits adopte une logique différente en matière d'urgence accessoire et en matière principale.

Comme on l'a vu précédemment, le contrôle de la qualification de l'ordre public en matière d'urgence accessoire a évolué avec le temps et au gré des textes: d'une présomption irréfragable de vérité, la qualification de l'autorité administrative est devenue banalement contrôlable.

C'est donc en matière principale que la qualification juridique des faits pose un problème majeur. Contrôlant les mesures de police, le juge camerounais se montre très réticent à contrôler la qualification juridique de faits, telle que faite par l'autorité administrative, comme cela a été constaté dans certaines décisions liées au contentieux des réunions et manifestations publiques. Dans l'affaire Jean Bosco Talla précitée, le juge judiciaire saisi s'en tient à la qualification de réunion publique, qui avait été faite sans doute par erreur ou par ignorance par l'autorité administrative, alors que le demandeur avait fait une déclaration pour manifestation publique. Cette requalification de l'autorité administrative avait été lourde de conséquences, puisque le juge judiciaire, exclusivement compétent pour le contentieux de l'interdiction des manifestations publiques s'était déclaré incompétent pour connaître du contentieux 
des réunions publiques qu'il considère comme relevant de la compétence du juge administratif. Dans l'affaire Moubarak Ibrahim Mbouobouo précitée, c'est l'inverse qui se produisit. Le juge administratif saisi conclut plutôt à une manifestation publique nonobstant le récépissé de déclaration et l'arrêté d'interdiction qui mentionnent tous une réunion publique. Sur cette qualification juridique erronée, le juge se déclare incompétent, renvoyant l'affaire devant le juge judiciaire ${ }^{77}$. Ce conflit négatif sur fond de qualification erronée nuit gravement au justiciable qui se voit sacrifié, mais surtout aboutit à une immunité de facto des mesures de police en ce sens que dans tous les cas, il constitue un empêchement péremptoire à leur contrôle sur le fond.

Par ailleurs, certains cas de qualification très approximative par l'autorité de police qui n'ont pas été portés devant le juge ${ }^{78}$ posent le problème de définition même de certaines notions. C'est le cas des réunions publiques, dont la difficulté de qualification par les autorités de police a souvent entraîné des conséquences dommageables pour les administrés ${ }^{79}$. La loi qualifie de publique toute réunion tenue dans un lieu public ou ouvert au public. Si le critère du lieu public est aisément compréhensible, celui du lieu ouvert au public peut prêter à équivoque. Il signifie qu'une réunion, même tenue dans un lieu privé, peut être qualifiée de publique, dès lors qu'elle est ouverte au public.

Le problème s'est posé relativement à une conférence organisée par un collectif de quatre partis politiques ${ }^{80}$ dans un lieu privé, en l'occurrence le siège de l'un d'entre eux, en vue du lancement du programme Stand for Cameroon, laquelle conférence a été qualifiée de réunion publique et interdite par le préfet de Yaoundé $4^{\mathrm{e}}$. S'agissait-il véritablement d'une réunion publique? Ce qui est sûr c'est que la tenue de la conférence au siège de l'un d'entre eux (lieu privé) par la coalition des partis sus-cités ne pouvait, à elle seule, suffire à écarter la qualification de réunion publique. Il s'agissait de déterminer si la condition de l'ouverture au public était satisfaite. À ce titre, il importait de convoquer le second critère de définition de la réunion publique qui renvoie au «rapport entre les organisateurs de la réunion et les personnes présentes $^{81}$ ». Cette condition devrait être considérée comme satisfaite si l'accès à la réunion n'est pas réservé à des personnes identifiées ou n'est pas fondé sur un lien personnel et individuel entre les organisateurs et les invités. Autrement dit, une réunion tenue dans un lieu privé devient publique si l'identification préalable des participants n'est ni prévue, ni possible ${ }^{82}$. En l'espèce, l'accès à la conférence était réservé à des journalistes nommément désignés à qui les organisateurs avaient envoyé des invitations personnelles. La condition de l'ouverture au public ne pouvait donc être considérée comme remplie. Il ne s'agissait donc pas d'une réunion publique. En revanche, la conférence de presse organisée par Abel Elimbi Lobe dans le Foyer Bazou (lieu privé), mais ouverte à tous (au public), pour annoncer son appel à la retraite du président Paul Biya, était bien une réunion publique ${ }^{83}$.

\subsubsection{La fragile reconnaissance du contrôle de la matérialité des faits}

Le contrôle de la matérialité des faits est celui par lequel le juge procède à la vérification de la véracité des faits incriminés. Contrôle de l'authenticité des motifs de la mesure de police, il ouvre la voie au contrôle des faits en permettant au juge d'aller au-delà d'une simple vérification de la légalité formelle de l'acte administratif. Le contrôle de la matérialité des faits est le prolongement de celui de la qualification juridique, car il est inconcevable qu'un « juge du fond s'abst[ienne] de vérifier la réalité des faits dont il doit apprécier la qualification juridique ${ }^{84}{ }$. 

favorable à un contrôle rigoureux de la matérialité des faits constituant le fondement de l'adoption de la mesure administrative ${ }^{85}$. Cependant, en matière d'ordre public, c'est seulement depuis l'affaire Mephou Philippe de $2011^{86}$, relative à la garde à vue administrative, que le juge a admis le principe du contrôle de la matérialité des faits. En l'espèce, accusé de «trouble à l'ordre public, violation des instructions des autorités administratives, non-respect des recommandations issues de la réunion du 28 octobre 1998 ", le sieur Mephou Philippe est alors gardé à vue par un arrêté du préfet du Wouri du 25 mars 1999. Saisi sur la légalité de cet acte, le juge affirmera : "attendu qu'il ne ressort du dossier aucune preuve ou même de simples indices justifiant les faits, motifs de l'arrêté ci-dessus attaqué. Attendu qu'ainsi l'arrêté qui n'est ni justifié, ni motivé est entaché d'excès de pouvoir et doit être annulé. » L'importance de cet arrêt n'est plus à démontrer lorsqu'on sait que le contrôle du juge est de moindre envergure dans les matières pour lesquelles l'autorité administrative jouit d'un pouvoir discrétionnaire ${ }^{87}$, à savoir lorsqu' «aucune norme juridique ne saurait déterminer [...] le choix de l'administration ${ }^{88} »$. Le juge empêche que la seule évocation des faits puisse être considérée comme fondant l'adoption de la mesure de police, sans vérification aucune. Le contrôle de la matérialité des faits peut donc être considéré, ainsi que c'est le cas en France depuis l'arrêt Camino ${ }^{89}$, comme constituant avec les autres contrôles (erreur manifeste, erreur de droit, détournement de pouvoir), le contrôle minimum du juge de l'excès de pouvoir ${ }^{90}$, c'est-à-dire la dimension irréductible de son contrôle. Par cet arrêté, le juge démontre qu'il n'est pas, comme on l'a souvent affirmé, lié par la qualification des faits telle qu'effectuée par l'autorité administrative ${ }^{91}$.

\subsection{L'inexistant contrôle du contenu des mesures de police}

La jurisprudence administrative témoigne d'un refus catégorique du juge de contrôler le contenu des mesures de police, c'est-à-dire d'une part l'adéquation de celles-ci aux situations de fait qui fondent leur adoption (2.2.1), et d'autre part l'étendue des pouvoirs des autorités de police (2.2.2).

\subsubsection{Le refus du contrôle de l'adéquation des mesures de police aux situations de fait}

Le contrôle de l'adéquation des mesures de police aux faits qui fondent son adoption se fait normalement à travers le contrôle de l'opportunité et celui de la proportionnalité des mesures de police. Ces deux dimensions du contrôle ${ }^{92}$, qui permettent au juge de s'assurer « de leur adéquation aux circonstances de temps et de lieu propres à chaque situation ${ }^{93}$ ", font défaut dans la jurisprudence du juge camerounais.

\subsubsection{L'exclusion du contrôle de l'opportunité des mesures de police}

La doctrine est divisée relativement à l'autonomie du contrôle de l'opportunité par rapport à celui de la légalité. Alors que pour certains, à l'instar de Daniel Chabanol, il

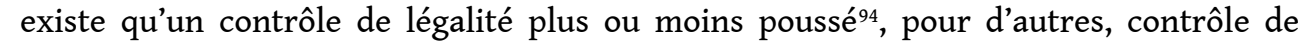
légalité et contrôle d'opportunité sont deux contrôles distincts qui procèdent de deux logiques et finalités différentes. Alors que l'un se limite au droit, l'autre plonge dans les circonstances non juridiques liées à l'adoption de l'acte ${ }^{95}$. 
l'administration, seule détentrice d'un pouvoir général d'appréciation. Ce choix du contrôle restreint peut se justifier, a fortiori, dans le cadre d'un pouvoir discrétionnaire comme celui dont l'autorité administrative est investie en matière d'ordre public. On sait, avec Charles Eisenmann, que la discrétionnalité vient de "l'incomplète ou l'imparfaite détermination de la réglementation [car] plus la réglementation est impérative et moins il y a de la place pour la discrétionnalité; moins elle est impérative, et plus le degré de discrétionnalité est grand ${ }^{101}$ ». L'ordre public n'étant pas véritablement circonscrit dans les textes, l'autorité de police se voit ainsi investi d'un pouvoir discrétionnaire important que le juge ne veut pas lui contester ${ }^{102}$. Car en contrôlant l'opportunité d'une mesure, notamment de police, le juge administratif imposerait en quelque sorte sa propre conception des circonstances de l'ordre public, puisque le contrôle de l'opportunité implique que le juge détermine, «à la suite de sa propre réappréciation des circonstances de l'affaire ${ }^{103}$ ", si la mesure critiquée convenait ou non. On aboutirait à «une administration par le juge ${ }^{104}$ »; celui-ci deviendrait alors, pour reprendre les termes de Stéphane Rials, un «super administrateur ${ }^{105} »$.

On peut toutefois questionner cette position du juge, puisque la question de l'opportunité constitue le point focal de la nécessité de la mesure d'ordre public. L'exclusion du contrôle de l'opportunité de la mesure de police peut être perçue comme une limite dans l'entreprise de conciliation des libertés et de l'ordre public que le juge doit effectuer. Pour René Chapus, pour une bonne protection des libertés face aux pouvoirs de l'administration, «la légalité n'importe pas seule. L'opportunité compte aussi ${ }^{106}$ ». Cette exclusion fait du choix de recourir à une mesure de police une décision souveraine incontestable, dont seule l'autorité de police apprécie le besoin, et qui ne peut être remise en cause. N'implique-t-elle pas simplement l'absence d'un contrôle du contenu même de la mesure d'ordre public ? Le refus du contrôle de la proportionnalité des mesures de police conforte cette hypothèse. 


\subsubsection{L'exclusion du contrôle de la proportionnalité des mesures de police}

51 Par le contrôle de la proportionnalité, le juge vérifie s'il existe une disproportion entre les motifs de fait et la mesure adoptée pour y remédier. Contrôle du « raisonnable » ou $\mathrm{du}$ " "bon sens" de l'appréciation de l'administrateur », il permet au juge de «faire une balance entre les moyens utilisés par l'administration, d'une part, et le but visé par l'administration, d'autre part ${ }^{107}$ ». Le juge met donc en rapport le contenu des mesures de police et les motifs et objectifs de celles-ci. L'importance d'un tel contrôle, dont le domaine d'élection par excellence est le rapport entre les mesures de police et la protection des libertés publiques ${ }^{108}$, se trouve dans le risque permanent d'une restriction inconsidérée de celles-ci par celles-là. Car concrètement, le juge doit se demander si une mesure de moindre importance du point de vue de ses effets restrictifs des libertés n'aurait pas été suffisante pour résoudre le problème.

Posé depuis le célèbre arrêt Benjamin en France ${ }^{109}$, où son principe est désormais solidement ancré, le contrôle de la proportionnalité tarde à faire ses premiers pas au Cameroun. Le juge administratif camerounais est constant dans son refus de procéder au contrôle de la proportionnalité des mesures de police. Ainsi, dans l'affaire Ekani André, le juge affirme n'avoir "pas qualité pour apprécier la gravité de la faute reprochée aux délinquants et la sanction à appliquer à cette faute. Que son rôle se borne à apprécier la légalité de la mesure prise $\mathrm{e}^{110} »$. Un peu plus récemment encore, ce constat s'est confirmé avec les affaires OCDH, CAP-Liberté et Kom Ambroise précitées où le juge affirme être «compétent que pour connaître si l'association dissoute tombe par ses agissements sous le coup de la loi ». Il était ici pourtant question de déterminer si la dissolution prononcée à l'encontre de ces associations était proportionnée aux faits qui leur étaient reprochés, notamment si une sanction inférieure telle que la suspension temporaire ou des sanctions à l'encontre des personnes ciblées n'étaient pas mieux indiquées. En bottant cette question en touche comme il la fait, le juge montre son intention de contrôler le moins possible l'administration dans son action et se pose en un complice coupable des dérives qui peuvent lui être imputées.

D'autres cas nécessitant le contrôle de la proportionnalité des mesures de police peuvent être signalés. Même s'ils n'ont pas été portés devant le juge, ils témoignent de la nécessité de censurer le recours aux interdictions générales et abstraites par les autorités de police. C'est le cas de l'arrêté du 15 janvier 2008 visant l'« interdiction des manifestations publiques dans la province du Littoral ${ }^{111}$ ", ou encore depuis 2010 de l'interdiction de «toute manifestation à caractère vindicatif et revendicatif sur toute l'étendue département du Mfoundi jusqu'à nouvel ordre» par le préfet dudit département ${ }^{112}$. C'est également le cas de l'interdiction de participation du parti politique Cameroon People's Party (CPP) au défilé de la fête nationale à Yaoundé depuis 2011, de l'interdiction de la mendicité et du petit commerce dans les rues et artères de la capitale par un arrêté du délégué à la communauté urbaine de Yaoundé du 17 juin 2003 (et réitérée, pour ce qui est de la mendicité, par le préfet du Mfoundi en juillet 2015). Ces interdictions générales et abstraites témoignent du défaut d'adéquation de la mesure «aux circonstances de temps et de lieu propre à chaque situation $^{113}$ ", qui constitue pourtant la marque de fabrique du contrôle du juge administratif français ${ }^{114}$. 


\subsubsection{L'oubli du contrôle de l'étendue des pouvoirs des autorités de police}

54 C'est surtout en matière de réunions et manifestations publiques que la question de l'étendue des pouvoirs de l'autorité de police se pose. À ce titre, les espèces Moubarak Ibrahim Mbombo et Jean Bosco Talla précitées, tout comme les cas MRC et autres ont révélé une nécessité impérieuse de procéder à la précision par le juge des limites des pouvoirs de l'autorité administrative en matière de réunions et manifestations publiques. Si les deux sont soumises au régime de la simple déclaration, sous réserve des réunions sur la voie publique soumises au régime de l'autorisation préalable, elles se distinguent $d u$ point de vue des pouvoirs reconnus à l'autorité administrative ${ }^{115}$. Alors que celle-ci peut interdire la tenue d'une manifestation publique déclarée en cas de menace de trouble à l'ordre public, un tel pouvoir ne lui est pas reconnu, relativement aux réunions publiques qui, une fois déclarées, ne peuvent être suspendues ou arrêtées que par le bureau organisateur ${ }^{116}$. Ceci peut s'expliquer par le fait que la tenue d'une réunion publique ne constitue pas une réelle menace à l'ordre public, en ce sens qu'elle se tient dans un lieu précis et clos, à la différence de l'organisation d'une manifestation publique qui a souvent lieu sur la voirie publique, comporte un nombre important de personnes parfois agitées et est souvent sous-tendue par un mobile politique ${ }^{117}$. La liberté des réunions publiques s'inscrit ainsi dans le cadre du régime répressif, plus libéral ${ }^{118}$, qui est la règle dans les pays de tradition libérale ${ }^{119}$. Le défaut de déclaration ne peut amener l'autorité administrative à interdire une réunion publique, ce défaut ne pouvant être sanctionné que par le juge pénal ${ }^{120}$. C'est dire que dans les cas Moubarak Ibrahim Mbombo, Abel Elimbi Lobe et a fortiori MRC et autres, le sous-préfet n'avait absolument aucun droit d'interdire la réunion déclarée par les organisateurs.

On pourrait néanmoins se demander si le pouvoir d'interdiction d'une réunion publique ne peut trouver son fondement dans la police administrative générale dont est investi le sous-préfet. Autrement dit, parce qu'il est le garant de l'ordre public, ne peut-il pas édicter toutes mesures nécessaires pour son maintien? Cette hypothèse qui est retenue en France ${ }^{121}$ doit être écartée au Cameroun : la réglementation relative aux réunions et manifestations publiques constituant une lex specialis, son application écarte le droit général relatif à l'ordre public. Ainsi, si le préfet est investi d'une mission générale de protection de l'ordre public, ses pouvoirs s'arrêtent aux portes des réunions publiques à propos desquelles la loi lui a expressément retiré toute possibilité d'agir.

Par ailleurs, deux questions se posent relativement au récépissé. Primo, la délivrance du récépissé est-elle une faculté ou une obligation pour l'autorité administrative ? La loi est claire sur la question, car tant pour les réunions que pour les manifestations publiques, la délivrance du récépissé de déclaration est de droit par l'autorité administrative, puisque la loi en fait une compétence liée. En conséquence, le refus de délivrance du récépissé de déclaration par l'autorité administrative dans l'affaire Jean Bosco Talla précitée est, à tout point de vue, constitutive d'un excès de pouvoir qui aurait dû être sanctionné par le juge, tout comme celui essuyé par les responsables du MRC en vue de l'organisation d'un meeting politique dans la ville de Bertoua, le 23 avril 2016.

Secundo, quel effet faut-il accorder au récépissé ainsi délivré par l'autorité administrative ? Est-ce ce document qui donne le droit de manifester ou de tenir une réunion? En affirmant dans l'affaire Moubarak Ibrahim Mbombo que « sont nulles et de 
nul effet, les dispositions de récépissé $\mathrm{n}^{\circ}$ 15/RD/C19/01/SP du 26 janvier 2005 accordant à Monsieur Sheik Ibrahim Moubarak, l'organisation d'une réunion publique le 8 février 2005 au lieu-dit Hôtel Arcade à Bonandjo ", l'arrêté d'interdiction laisse entendre que c'est le récépissé qui donne droit à la tenue de la réunion publique. Ce qui est loin d'être le cas, car c'est plutôt la formalité de déclaration qui est créatrice de droit à manifester ou de tenir une réunion publique, le récépissé n'étant plus alors qu'un simple élément de preuve, qui constate cette volonté.

Comme on peut le remarquer à travers sa jurisprudence, le juge administratif camerounais procède à un contrôle très minimum de l'utilisation par l'administration des mesures de police. Sa jurisprudence en la matière montre qu'il est un juge conservateur et prudent, peu tenté par la hardiesse. Dans certains cas, il se présente même comme un juge servile et complice d'une administration considérée comme le bras séculier d'un pouvoir politique omniprésent désireux de perpétuer insidieusement la dynamique autoritaire pourtant officiellement révolue ${ }^{122}$. En conséquence, le contrôle timoré du juge sur les actes de police donne de lui l'image d'un juge plus protecteur des prérogatives de l'administration que soucieux de garantir les libertés et fait de lui « le dernier bouclier d'un système arbitraire ${ }^{123}$ ».

\section{NOTES}

1. Étienne Picard, "L'influence du droit communautaire sur la notion d'ordre public ", Actualité juridique, droit administratif, 20 juin 1996, nº spécial, p. 56.

2. Gérard Cornu, Vocabulaire juridique, Paris, PUF, «Quadrige», 2002, p. 619. Gérard Cornu définit l'ordre public en droit privé comme un «terme servant à caractériser certaines règles qui s'imposent avec une force particulière [...] et par extension [...] l'ensemble des règles qui présentent ce caractère ».

3. Étienne Picard, op. cit., p. 57.

4. En zone CEMAC, la réserve d'ordre public est contenue dans les art. 16, 27(a) et 98 de la Convention régissant l'Union économique de l'Afrique centrale (UEAC) adoptée le 25 juin 2008 à Yaoundé (Cameroun) qui remplace celle précédemment en vigueur du 16 juillet 1996, adoptée à Libreville au Gabon.

5. Pascale Deumier, Thierry Revet, «Ordre public », dans Denis Alland et Stéphane Rials (dir.), Dictionnaire de la culture juridique, Paris, Lamy-PUF, « Quadrige », 2003, p. 1119.

6. Jacques Foyer, «La convention européenne des droits de l'Homme et l'exception d'ordre public international ", dans Mélanges Raymond Goy. Du droit interne au droit international. Le facteur religieux et l'exigence des droits de l'homme, Rennes, PUR, 1998, p. 335. L'évocation de l'ordre public conduit à éviter l'application des « lois étrangères jugées trop choquantes ou trop différentes ».

7. Alain Pellet, "Vive le crime! Remarques sur les degrés de l'illicite en droit international ", dans Alain Pellet (dir.), Le droit international à l'aube du xxI siècle. Réflexions de codificateurs, New York, Nations unies, 1997, p. 307-314. 
8. Paul Bernard, La notion d'ordre public en droit administratif français, Paris, LGDJ, 1962, p. 169.

9. Martine Lombard, Droit administratif, Paris, Dalloz, 1997, p. 170.

10. Maurice Hauriou, Précis de droit administratif et de droit public, $12^{\mathrm{e}}$ éd., Sirey, 1933 , p. 549. Jean Waline, Droit administratif, $2^{\mathrm{e}}$ éd., Paris, Dalloz, 2008, p. 325.

11. CE, 18 décembre 1959, Société Les films Lutétia, Rec. 693; CE, 27 octobre 1995, Commune de Morsang-sur-Orge, et CE, 27 octobre 1995, Ville d'Aix-en-Provence, Rec. 372.

12. La Constitution est l'expression parfaite de cette inconstance dans la référence à l'ordre public. Voir préambule, $\S 3,4^{\mathrm{e}}$ point, d'une part et les art. 58,59 et $60 \mathrm{du}$ corpus d'autre part. L. $n^{\circ} 2006-022$, 29 déc. 2006, art. 30 fixant l'organisation et le fonctionnement des tribunaux administratifs reprend cette confusion en mettant côte à côte l'ordre public, la sécurité publique et la tranquillité publique.

13. Ordonnance de référé $n^{\circ} 37 / \mathrm{OR} / \mathrm{CAB} / \mathrm{PCA} / \mathrm{CS} / 2003-2004$, Affaire Nguessie Joseph c. État du Cameroun (CUY).

14. Const., préambule, $\S 3,13^{\mathrm{e}}$ point: «Nul ne peut être inquiété en raison de ses origines, de ses opinions ou croyances en matière religieuse, philosophique ou politique sous réserve du respect de l'ordre public et des bonnes mœurs." Certaines lois instituant des polices administratives spéciales ont également repris cette formule, à l'instar de la police administrative en matière de communication sociale (L. $\mathrm{n}^{\circ}$ 90-052, art. 17, portant liberté de communication sociale au Cameroun); de la police administrative en matière de liberté d'association (L. $\mathrm{n}^{\circ}$ 90-053, art. 3, portant liberté d'association); et de la police des réunions publiques (L. $\mathrm{n}^{\circ}$ 90-055, art. 5, portant régime des réunions et des manifestations publiques).

15. Voir les affaires Société Les Films Lutétia, Commune de Morsang-sur-Orge et Ville d'Aixen-Provence précitées.

16. Bernard Stirn, "Ordre public et libertés publiques", Actes du colloque sur l'ordre public, organisé par l'Association française de philosophie du droit, Paris, Fondation JeanJaurès, 17 et 18 septembre 2015, p. 1 .

17. René Demogue, Les notions fondamentales du droit privé. Essai critique, Paris, A. Rousseau, 1911, p. 146, «L'ordre public, ce sont les idées auxquelles la société s'est très nettement arrêtée, repoussant la liberté sur ce point puisqu'elle croit tenir la vérité ».

18. CC, décision $\mathrm{n}^{\circ}$ 85-187 DC, 25 janvier 1985, Loi relative à l'État d'urgence en NouvelleCalédonie et dépendances.

19. Étienne Picard, La notion de police administrative, t. 1, Rouen-Paris, Publications de l'université de Rouen-LGDJ, 1984, p. 541. On lira, dans le même sens, Charles-Édouard Minet, Droit de la police administrative, Paris, Vuibert, 2007.

20. Thomas Dumortier, L'ordre public. Essai sur quelques usages contemporains d'un standard classique, thèse de doctorat en droit, université Paris Nanterre, 2010, p. 133-140. L'auteur se contente de présenter les thèses en présence, sans prendre position.

21. Ibid. p. 232.

22. Philippe Terneyre, «Les adaptations aux circonstances du principe de constitutionnalité : contribution du Conseil constitutionnel à un droit constitutionnel de la nécessité », RDP, 1987, p. 1514. 
23. La Constitution camerounaise évoque la notion d'ordre public à cinq reprises, dont deux fois dans le préambule et trois fois dans le corpus lui-même. Il s'agit donc d'une constitutionnalisation textuelle et directe de l'ordre public, qui tranche avec le choix de la constitutionnalisation indirecte [l'ordre public est mentionné par l'art. 10 de la décl. de 1789, lui même visé par le préambule de la Constitution de 1958 auquel le Cons. const. a reconnu la valeur constitutionnelle dans sa décision $\mathrm{n}^{\circ}$ 71-44 DC du 16 juillet 1971, Liberté d'association] et jurisprudentielle [dans sa décision $\mathrm{n}^{\circ}$ 82-141 DC, 27 juillet 1982, Loi relative à la communication audiovisuelle, le Cons. Const. élève l'ordre public au rang de principe à valeur constitutionnelle] fait par la France.

24. Agnès Makougoum, Ordre public et libertés publiques en droit public camerounais. Contribution à l'étude de la construction de l'État de droit au Cameroun depuis 1990, thèse de doctorat, université de Yaoundé II, 2013-2014.

25. L'art. 9 de l'ord. $n^{\circ} 72 / 6$ du 26 août 1972 fixant l'organisation de la Cour suprême est la parfaite illustration de cette option. Après avoir reconnu à la faveur du juge administratif «l'ensemble du contentieux administratif à l'encontre de l'État, des collectivités publiques et établissements publics ", il procédait à une énumération limitative avant de reconnaître aux tribunaux de droit commun le droit de connaître, " conformément aux règles de droit privé, de tout autre action ou litige, même s'il met en cause les personnes morales énumérées au paragraphe premier ». Ce schéma a été repris mutatis mutandis par la loi de 2006 fixant l'organisation et le fonctionnement des tribunaux administratifs précitée, qui d'une part reconnaît le juge administratif comme le juge de droit commun du contentieux administratif (art. 2(1) et 14(1)), et d'autre part procède à une énumération limitative des chefs de compétence qui lui sont (art. 2(3)) avant de conférer au juge judiciaire tout autre litige mettant en cause les personnes morales de droit public (art. 3).

26. Le juge judiciaire est titulaire de plusieurs chefs de compétence qui lui sont attribuées par la loi camerounaise: il connaît de l'emprise, de la voie de fait, de l'interprétation et de l'appréciation de la légalité des actes administratifs. Sur la question, lire Célestin Keutcha Tchapnga, Précis de contentieux administratif au Cameroun. Aspects de l'évolution récente, Paris, L'Harmattan, 2013, p. 105.

27. CS/CA, jugement $n^{\circ} 94 / 2011 / C A / C S$ du 23 mars 2011, Moubarak Ibrahim Mbombo c. État du Cameroun.

28. Ce deuxième aspect sera examiné plus loin. Voir infra. 2.2.2. L'oubli du contrôle des pouvoirs des autorités de police.

29. Agnès Makougoum, op. cit., p. 434.

30. Cette position a d'ailleurs été adoptée par le juge judiciaire dans sa décision rendue en 2013 à l'occasion de l'affaire Jean Bosco Talla.

31. Pierre-Paul Tchindji, « Le sort de la liberté dans la législation camerounaise sur la liberté de communication sociale », AFSJP, t. 2, p. 77-99, spéc. p. 87.

32. Ordonnance de référé $\mathrm{n}^{\circ} 13 / \mathrm{OR} / \mathrm{CS} / \mathrm{PCA} / 90-91$, Le Messager c. État du Cameroun, 25 avril 1991.

33. Bernard Raymond Guimdo Dongmo, "Note sur l'Ordonnance de référé $n^{\circ} 13 / O R /$ PCA/90-91 du 25 avril 1991 Affaire journal Le Messager c. État du Cameroun ", Juridis Infos $\mathrm{n}^{\circ} 17$, janvier-février-mars 1994, p. 55. 
34. Jean Essomba Tsama, La répartition des compétences entre le juge administratif et le juge judiciaire en matière de libertés publiques au Cameroun, mémoire de DEA, université de Yaoundé II, 2005-2006, p. 67.

35. Laëtitia Allemand et Jean-Michel Oullion, Les grandes questions des médias. Enjeux et stratégies des médias contemporains, Paris, L'Étudiant, 2005, p. 130.

36. L. n $^{\circ}$ 96-04, 4 janv. 1996, art. 17(2), modifiant certaines dispositions de la loi de 1990 précitée sur la communication sociale.

37. Jean Essomba Tsama, La répartition des compétences entre le juge administratif et le juge judiciaire en matière de libertés publiques au Cameroun, op. cit., p. 66 et 67.

38. C'est seulement en 2006 que la loi fixant organisation et fonctionnement des tribunaux administratifs précitée instituera les tribunaux administratifs en raison d'un tribunal par région.

39. Ordonnance de référé du 4 juillet 1997, Affaire Mutations c. État du Cameroun.

40. Cour d'appel du Centre, arrêt du 24 octobre 1997, Affaire Mutations c. État du Cameroun.

41. Jean Essomba Tsama, op. cit., p. 66 et 67.

42. Il s'agissait ainsi des interlocutory applications qui peuvent prendre la forme des mandatory ou prohibitory injunctions. Sur ces procédures, lire Michael A. Yanou, Practice and Procedure in Civil Matters in the Courts of records in Anglophone Cameroon, Bamenda, Langaa Research and Publishing, CIG, 2015, p. 136.

43. Une précision s'impose ici. Certes y a-t-il eu une introduction du droit administratif dans la zone anglophone du pays dès la Constitution fédérale de 1961, dont l'art. 33 attribue l'ensemble du contentieux administratif national à la Cour fédérale de justice, et plus tard, par les lois n ${ }^{\circ} 65-\mathrm{LF}-29$ du 19 novembre 1965 et n ${ }^{\circ}$ 69-LF-1 du 14 juin 1969, cette dernière créant au sein de la Cour fédérale de justice, en matière administrative, une Assemblée plénière, juge d'appel, et deux sections dans le ressort des deux États fédérés (Yaoundé et Buea) statuant en premier ressort. Après les carences observées dans cette organisation et notamment la difficulté pour la section administrative du Cameroun occidental de maîtriser le contentieux administratif, cette introduction procédera d'une tout autre logique au lendemain de la réunification, avec le remplacement des sections administratives par la chambre administrative ayant son siège à Yaoundé (sur cette question, voir Célestin Sietchoua Djuitchoko, "Souvenir de la Common Law et actualité juridique du droit administratif dans les provinces anglophones du Cameroun ", Revue générale de droit, vol. 27, n 3, sept. 1996, p. 357-374, spéc. p. 365). Cependant, il faut dire que malgré cette consécration théorique de l'introduction du droit administratif dans le Cameroun anglophone, la pratique révélait de fortes réticences de la part des juges de l'ordre judiciaire, qui continuaient de connaître des litiges relevant du contentieux administratif. Cet état de fait va changer avec la mise en place récente et progressive des tribunaux administratifs créés par le texte constitutionnel de 1996. Cette distinction rentrait en droite logique avec l'art. 38 de la Constitution unitaire de 1972 qui, tout en mettant officiellement un terme au fédéralisme institué depuis 1961, le reconduisait implicitement dans des matières non harmonisées en permettant l'application des lois en vigueur dans les ex-États du Cameroun oriental francophone et du Cameroun occidental anglophone. Cette reconduction sera d'ailleurs reprise par le texte constitutionnel du 18 janvier 1996 en son art. 68. 
44. Olivier Fandjip, "Les décrets des 15 mars et 18 avril 2012 ou l'effectivité d'une réforme et la résurgence des problématiques de l'accès et de l'autonomie de la justice administrative au Cameroun ", Revue juridique et politique des États francophones, $\mathrm{n}^{\circ} 1$, 2013, p. 60-78.

45. Art. 2(2)(e).

46. Sur cette règle d'interprétation, lire Vinvent Correia, «L'adage lex specialis derogat generali: Réflexions générales sur sa nature, sa raison d'être et ses conditions d'application ", www.academia.edu.

47. Célestin Sietchoua Djuitchoko, «Souvenir de la Common Law... », art. cité.

48. Simone Goyard-Fabre, "L'innovation dans le champ juridique ou le passé recomposé", Revue de métaphysique et de morale, vol.83, $\mathrm{n}^{\circ}$ 3, 2014, p.311-326, spéc. p. 316.

49. Jacques Petit, "Les armes du juge administratif dans la protection des libertés fondamentales ", dans Gweltaz Éveillard (dir.), Actes du colloque : La guerre des juges aurat-elle lieu? Analyse comparée des offres du juge administratif et du juge judiciaire dans la protection des libertés fondamentales, université de Rennes 1, 3 avril 2015, 2016, www.revuegeneraledudroit.eu. Jacques Petit considère les procédures d'urgence comme de nouvelles armes légales dont le juge administratif français est doté dans son rôle de protecteur des libertés publiques face à l'administration.

50. Bernard Pacteau, Contentieux administratif, Paris, PUF, 1989, p. 235. Bernard Pacteau le définira de manière métaphorique comme «la détention provisoire de l'acte non encore condamné, mais déjà inculpé ».

51. Bernard Raymond Guimdo Dongmo, Lejuge administratif camerounais et l'urgence: recherches sur la place de l'urgence dans le contentieux administratif camerounais, thèse d'État, université de Yaoundé II-Soa, 2004, p. 65.

52. Voir art. 27 et 30.

53. Cette procédure se rapproche davantage du référé conservatoire en France. Sur la question, voir Jean-Luc Rongé, «L'évolution du référé administratif », Journal du droit des jeunes, vol. 207, $\mathrm{n}^{\circ}$ 7, 2001, p. 27-29.

54. Célestin Keutcha Tchapnga, «Le régime du sursis à exécution dans la jurisprudence administrative camerounaise ", Juridis périodique $\mathrm{n}^{\circ}$ 38, avril-mai-juin 1999, p. 83-92, spéc. p. 91.

55. «Attendu que la décision ne concerne ni l'ordre public, ni la sécurité, ni la tranquillité publique.»

56. . Célestin Keutcha Tchapnga, « Le régime du sursis... », art. cité.

57. Voir l'ordonnance de référé $\mathrm{n}^{\circ} 30 / \mathrm{OR} / \mathrm{PCA} / \mathrm{CS} / 2001-2002$, du 10 avril 2002, Union des Populations du Cameroun (UPC-Kodock) c. État du Cameroun (MINAT) et Union des Populations du Cameroun (UPC tendance Hogbe Nlend). Le même constat est fait en matière disciplinaire: Ordonnance sursis CS/CA, 7 décembre 2000, Mama Biloa Sandrine c. Université de Ngaoundéré. Célestin Keutcha Tchapnga, «La reforme attendue du contentieux administratif au Cameroun », Juridis Périodique, $\mathrm{n}^{\circ}$ 70, avril-mai-juin 2007, p. 28.

58. Jacques Bipele Kemfouedio et Olivier Fandjip, « Le nouveau procès administratif au Cameroun : réflexion sur le recours gracieux en matière d'urgence », RIDC, vol. 64, $n^{\circ} 4$, 2012, p. 973-993. 
59. Agnès Makougoum, op. cit., p. 223.

60. L'autorité destinataire du recours gracieux préalable a évolué dans le temps. Du «ministre compétent» sous l'ordonnance $n^{\circ} 76 / 06$ du 26 août 1972 portant organisation et fonctionnement de la Cour suprême (art.12(1)), notion qui avait dérouté à la fois juge et justiciable, on est passé à la notion plus claire et précise d'« autorité auteur de l'acte attaqué ou [...] celle statutairement habilitée à représenter la collectivité publique ou l'établissement public en cause ». Lire Célestin Keutcha Tchapnga, op. cit., p. 161-162.

61. Voir art. 17 de la loi de 2006 fixant l'organisation et le fonctionnement des tribunaux administratifs précitée.

62. Pour le contentieux de la légalisation des partis politiques, voir les ordonnances $\mathrm{n}^{\circ}$ 02/O/PCA/CA/CS du 16 décembre 1992, Union des Populations du Cameroun - Manidem (UPC-Manidem) c. État du Cameroun, et $n^{\circ}$ 28/CS/PCA/91-92 du 23 septembre 1992 Union Nationale Camerounaise (UPC) (Stanley Akwote Akondi) c.État du Cameroun; pour le contentieux de la dissolution des associations, voir les ordonnances $n^{\circ} 19 / \mathrm{PCA} / \mathrm{CS} d u$ 26 septembre 1991, Organisation camerounaise des droits de l'homme (OCDH), $\mathrm{n}^{\circ}$ 20/PCA/CS du 26 septembre 1991, Kom Ambroise et n²1/O/PCA/CS du 26 septembre 1991, Comité d'action populaire pour la liberté et la démocratie (Cap-Liberté); pour le contentieux des réunions et manifestations publiques, voir l'affaire Moubarak Ibrahim Mbombo précitée ; pour le contentieux de la presse, voir les affaires Le Messager et Mutations précitées.

63. Voir successivement les art. 17(3) de la loi de 1996 modifiant certaines dispositions de la loi de 1990 précitée ; art. 8(3) de la loi de 1990 fixant le régime des réunions et manifestations publiques précitée; art. 13 alinéa 3 de la loi de 1990 portant liberté d'association précitée; art. 8(3) de la loi $n^{\circ} 90 / 056$ relative aux partis politiques et art. 22(3) de la loi $n^{\circ} 99 / 014$ du 22 décembre 1999 régissant les organisations non gouvernementales.

64. Bernard Raymond Guimdo Dongmo, op. cit., p. 277. Il constate la lenteur légendaire du juge administratif camerounais qui constitue une "source de gâchis économique et social, et nuit à l'image de la justice administrative qui est discréditée, les justiciables [...] exaspérés, les avocats [...] découragés ».

65. Olivier Fandjip, Les juridictions administratives et le temps : cas du Cameroun et du Gabon, mémoire de DEA, université de Dschang, Cameroun, 2009.

66. Patrice Rolland, La protection des libertés publiques en France, Paris, Dalloz, 1995, p. 33.

67. Bernard Raymond Guimdo Dongmo, op. cit., p. 210.

68. La « légalité » étant ici prise dans son sens élargi comme « la soumission à la loi, soumission d'un acte juridique, mais aussi d'une activité matérielle à la norme juridique, elle-même élargie au-delà de la loi formelle à l'ensemble de la normativité ». Voir Yves Gaudemet, " Principe de légalité », dans Denis Alland et Stéphane Rials (dir.), op. cit., p. 918.

69. Bertrand Seiller, Droit administratif. 2: L'action administrative, $2^{\mathrm{e}}$ éd., Paris, Flammarion, 2005, p. 236.

70. On signalera simplement à ce sujet que le juge a posé le principe de la théorie de la connaissance acquise dans l'affaire $O C D H$, où il considère comme valable l'acte de dissolution non notifié, mais dont l'intéressé a eu connaissance par communiqué radio et ensuite à partir de ses propres initiatives. Sur la question, lire Célestin Keutcha Tchapnga, Célestin Sietchoua Djuitchoko, «Commentaire de l'arrêt de la Cour Suprême 
du Cameroun, Chambre Administrative, Jugement $\mathrm{n}^{\circ} 29$ du 3 mai 1990, Mbarga Symphorien contre État du Cameroun (MFPCE) », Afrilex, $n^{\circ}$ 1, 2000.

71. Nadine Poulet-Gibot Leclerc, Droit administratif: sources, moyens, contrôles, $3^{\mathrm{e}}$ éd., Rosny-sous-Bois, Bréal, 2007, p. 246.

72. René Chapus, Droit administratif général, $15^{\mathrm{e}}$ éd., Paris, Montchrestien, 2001, p. 1047.

73. Bertrand Seiller, op. cit., p. 239. Nadine Poulet-Gibot Leclerc, op. cit., p. 247.

74. Nadine Poulet-Gibot Leclerc, ibid.

75. CE, 4 avril 1914, sieur Gomel, Rec., p. 488.

76. René Chapus, Droit administratif général, op. cit., p. 1043-1044.

77. Voir supra. 1.1.1. L'imprécise compétence en matière de contentieux relatif aux réunions et manifestations publiques.

78. Ce qui dénote de l'esprit peu procédurier des Africains décrié par Keba Mbaye, Les droits de l'homme en Afrique, $2^{\mathrm{e}}$ éd., A. Pedone, Paris, 2002 p. 86.

79. Commission nationale des droits de l'homme et des libertés (CNDHL), Rapport sur l'état des droits de l'homme au Cameroun, 2004.

80. Il s'agissait du Mouvement pour la renaissance du Cameroun (MRC), du Cammeroon People's Party (CPP), de l'Union des populations du Cameroun-Les Fidèles (UPC-Les Fidèles) et du parti de l'Univers.

81. Guillaume Plas, Le droit de réunion, le maintien de l'ordre public et les autorités de police administrative, Heule, UGA, 1999, p. 131.

82. Olivier Gohin, «Liberté d'expression, liberté de réunion, police administrative et ordre public: l'affaire Dieudonné » Note sous tribunal administratif de Nantes, ordonnance, RFDA, 9 janvier 2014, p. 87.

83. Son interdiction par l'autorité administrative, tout comme celle de la conférence du $\mathrm{MRC}$ et autres (à considérer même qu'elle soit une réunion publique), est constitutive d'excès de pouvoir dès lors que la loi ne lui en reconnaît pas un tel pouvoir en matière de réunion publique. Voir infra. 2.2.2. L'oubli du contrôle des pouvoirs des autorités de police.

84. René Chapus, op. cit., p. 1046.

85. Dans l'affaire Ekong Yves Adolphe, il affirme déjà que « le rôle de la juridiction administrative est de rechercher si les faits invoqués sont matériellement exacts, si ces faits justifient une sanction administrative, si la procédure disciplinaire a été régulière, si enfin les droits de la défense ont bien été sauvegardés » (jugement $n^{\circ} 27 / C C A d u$ 27 novembre 1953). On retrouvera la même affirmation dans les affaires Toko Daniel ( $n^{\circ}$ 134/CFJ/SCAY du 30 avril 1968), Biba Théophile (n 53/CFJ/SCAY du 25 mars 1969), Mbime Joël (CCA, décision $\mathrm{n}^{\circ} 35$ du 10 août 1950) et Nguenyang Jean ( ${ }^{\circ} 131 / \mathrm{TE} \mathrm{du}$ 23 décembre 1960).

86. Jugement $n^{\circ}$ 134/2011/CA/CS du 13 avril 2011.

87. Nadine Poulet-Gibot Leclerc, op. cit., p. 247.

88. Bertrand Seiller, op. cit., p. 235.

89. CE, 14 janvier 1916, Camino, Rec. 15.

90. Bertrand Seiller, op. cit., p. 235.

91. Agnès Makougoum, op. cit., p. 383. 
92. Contrôle de proportionnalité et contrôle d'opportunité sont intimement liés. Gregory Kalflèche se demande même si le juge ne procède pas à un contrôle d'opportunité lorsqu'il contrôle la proportionnalité des décisions administratives. Voir Gregory Kalflèche, "Le contrôle de proportionnalité exercé par les juridictions administratives ", Les Petites Affiches, n 46, spécial, 5 mars 2009, p. 46-53, spéc. p. 46.

93. Bernard Stirn, «Ordre public et libertés publiques », art. cité, p. 7.

94. Daniel Chabanol, "Contrôle de légalité et libertés de l'administration", AJDA, janvier 1984, p. 15. On retrouvera cette position chez Yves Gaudemet (art. cité, p. 917), qui considère tout contrôle fait par le juge comme un contrôle de légalité lato sensu.

95. Agnès Makougoum, op. cit., p. 407.

96. Marc Reglade, « Contrôle juridictionnel de l'opportunité », RDP, 1925, p. 414.

97. André de Laubadère, «Le contrôle juridictionnel du pouvoir discrétionnaire dans la jurisprudence récente du Conseil d'État», dans Mélanges Waline, Paris, LGDJ, 1974, p. 531.

98. S'il s'est, dès le début, refusé le droit de contrôler l'opportunité des décisions administratives dans les affaires Gomel et Camino précitées, dans certains cas comme les affaires Ville nouvelle Est et Sainte-Marie de l'Assomption, le juge administratif français s'offre le droit de questionner certains choix de l'autorité administrative (CE, 28 mai 1971, Ville nouvelle Est, Rec. 409 ; Ass., 20 octobre 1972, Société civile Sainte-Marie de l'Assomption, Rec. 657).

99. Dans l'affaire Ekong Yves Adolphe précitée, il affirme que «le rôle de la juridiction administrative est de rechercher si les faits invoqués sont matériellement exacts, si ces faits justifient une sanction administrative [...] ».

100. On verra les affaires Thabene Hubert (jugement $n^{\circ} 32 / C C A$ du 11 août 1950) ; Nkongo Mayo (jugement $n^{\circ}$ 243/CCA du 10 juillet 1953); Mikoma Albert (jugement $n^{\circ} 263 /$ CCA du 27 novembre 1953); Mouchili Isaac (jugement $n^{\circ}$ 294/CCA du 27 mars 1954).

101. Charles Eisenmann, Cours de droit administratif, t. 2, LGDJ, Paris, 1983, p. 429.

102. Bertrand Seiller abonde dans ce sens lorsqu'il dit, à ce sujet, que le contrôle normal n'est mis en œuvre par le juge que lorsque celui-ci «estime que le bloc de légalité n'accorde pas ou peu de pouvoir discrétionnaire à l'administration » (op. cit., p. 239).

103. André de Laubadère, op. cit., p. 533.

104. Raymond Odent, "Contentieux administratif», Cours à l'Institut d'études politiques de Paris 1976-1981, Les Cours du droit, p. 2006-2007.

105. Stéphane Rials, Le juge administratif français et la technique du standard. Essai sur le traitement juridictionnel de l'idée de normalité, Paris, LGDJ, 1980 p. 249.

106. René Chapus, op. cit., p. 1056.

107. Gregory Kalflèche, op. cit., p. 46.

108. Jean-Paul Costa «Le principe de proportionnalité dans la jurisprudence du Conseil d'État », AJDA, 1988, p. 435.

109. CE, 19 mai 1933, Benjamin, Rec. p. 541.

110. Jugement $n^{\circ} 323 / \mathrm{CCA}$ du 10 décembre 1954, Ekani André. On retrouvera cette position dans les affaires Otyam Belinga Martin (jugement $\mathrm{n}^{\circ} 329 / \mathrm{CCA}$ du 10 décembre 1954) et Lembegue Telesphore (jugement $n^{\circ} 334 / C C A$ du 15 mars 1955). 
111. Mesure qui avait été adoptée à l'époque en vue d'éviter toute manifestation tendant à dénoncer les velléités de modification constitutionnelle par le pouvoir, pour faire sauter le verrou de la limitation des mandats présidentiels et permettre au président de la République de se représenter.

112. Voir la lettre préfectorale $\mathrm{n}^{\circ} 0000119 / \mathrm{L} / \mathrm{J} 06.03 / \mathrm{Sp}$ du 8 novembre 2010. Plusieurs manifestations seront ainsi interdites sur ce seul fondement, notamment la manifestation organisée le 11 novembre 2010 par la Centrale syndicale du secteur public (CSP) en vue de faire part aux autorités de certaines de leurs réclamations, la marche paysanne organisée le 31 mai 2011 à l'initiative de l'Association citoyenne de défense des intérêts collectifs (ACDIC), les manifestations prévues dans le cadre des activités politiques du Parti des démocrates camerounais (PDC) en juillet 2011 ou encore la manifestation organisée en marge de la journée internationale de la femme de l'année 2015 par le parti politique Social Democratic Front (SDF), en vue de la protestation sur l'approvisionnement en eau et en électricité dans l'arrondissement de Yaoundé 4.

113. Bernard Stirn, op. cit., p. 6.

114. Voir CE, 22 juin 1951, Daudignac, Rec. 362 (sur l'interdiction générale de l'activité de photographe-filmeur); CE, Ass. 24 janvier 1975, Ministre de l'information c. société Rome-Paris Films, Rec. 57 (sur l'interdiction générale d'exploitation d'un film en France); CE, 2 août 2001, Préfet de Vaucluse et CE, 10 août 2001, Commune d'Yerres (sur l'interdiction générale de sortie des mineurs non accompagnés); CE, 9 juillet 2003, Laurent L. c. commune de Prades (sur l'interdiction générale de mendicité).

115. La déclaration préalable renvoie au régime dans lequel l'autorité administrative exige simplement de l'individu qu'il l'informe de son intention de tenir certaines activités. Quant à l'autorisation préalable, elle désigne l'acte par lequel l'autorité administrative conditionne l'activité à son intervention positive, à savoir que c'est elle qui permet à une personne d'exercer une activité ou de jouir de certains droits dont l'exercice et la jouissance sont subordonnés à son obtention. Sur ces notions, lire Xavier Latour et Bertrand Pauvert, Libertés publiques et droits fondamentaux, Paris, Studyrama, 2006, p. 74.

116. Art. 5 et 8 de la loi de 1990 portant régime des réunions et des manifestations publiques précitée.

117. Gabriel Babineau, «La manifestation : une forme d'action collective ", Les Cahiers de droit, vol. 53, n 4, déc. 2012, p. 761-792.

118. Claude Leclercq, Libertés publiques, $4^{e}$ éd., Paris, Litec, p. 146.

119. Maurice Kamto, Droit de la presse au Cameroun, Yaoundé, Fondation Friedrich Ebert au Cameroun, 1993, p. 16.

120. Art. 9 et 10 de la loi de 1990 portant régime des réunions et des manifestations publiques précitée.

121. En France, toute réunion publique est «susceptible d'une mesure d'interdiction, au titre de la police municipale, en tant que police générale ", sur le fondement de l'art. L. 2212-2 du code général des collectivités territoriales, selon lequel « la police municipale a pour objet d'assurer le bon ordre, la sûreté, la sécurité et la salubrité publique. Elle comprend notamment: [...] Le maintien du bon ordre dans les endroits où il se fait de grands rassemblements d'hommes, tels que les [...] spectacles [...]». Voir Olivier Gohin, art. cité. 
122. Bernard Raymond Guimdo Dongmo, op. cit., p. 488. Analysant les décisions, $O C D H$, Kom Ambroise et CAP-Liberté précitées, B. R. Guimdo Dongmo parlera de jurisprudence plus politique que juridique.

123. Agnès Makougoum, op. cit., p. 427.

\section{RÉSUMÉS}

De l'avis général, la libéralisation de la vie politique en Afrique au début des années 1990 s'est caractérisée, au Cameroun en particulier, par la mise en place progressive d'un dispositif législatif et institutionnel qui prend mieux en compte les libertés publiques. Le présent article vise à questionner ce postulat et à déterminer jusqu'à quel degré il est vérifié. À ce titre, s'il est évident que le législateur camerounais a, dès le début de la décennie 1990 institué un cadre institutionnel mieux adapté pour la garantie des libertés publiques face aux pouvoirs de police de l'administration, il reste que la conciliation entre l'ordre public et les libertés se fait encore, de nos jours, au détriment de celles-ci. Ce déséquilibre se manifeste aussi bien à travers le caractère mitigé de l'encadrement législatif du contrôle du juge que dans les choix jurisprudentiels du juge. Sur l'encadrement législatif du contrôle, une double tendance se dégage. D'une part, la rédaction souvent tatillonne des textes laisse peu de lisibilité sur la juridiction compétente pour connaître des actes de police dans certaines matières précises, ce qui débouche sur un conflit de compétence qui lui-même est susceptible d'aboutir à un déni de justice. D'autre part, on note une libéralisation progressive de la procédure contentieuse en matière de mesures de police qui se caractérise aussi bien par la levée partielle de l'immunité provisoire des mesures de police et l'institution de certaines garanties procédurales propres à assurer l'efficacité du contrôle du juge. Quant aux choix jurisprudentiels, ils témoignent de la réticence du juge de procéder à un contrôle étendu des mesures de police. Cette réalité se traduit par l'insuffisant contrôle des faits justifiant les mesures de police, qu'il s'agisse du contrôle de leur qualification juridique ou celui de leur matérialité, d'une part, et par le refus du contrôle du contenu des mesures de police, à savoir celui de leur opportunité et celui de leur proportionnalité, d'autre part.

There is general agreement that the liberalization of political life in Africa in the early 1990s has been characterized, in Cameroon in particular, by the progressive establishment of a legislative and institutional framework that takes better account of public freedoms. The purpose of this article is to question this assumption and to determine to what extent it is verified. In this respect, while it is clear that the Cameroonian legislator has, since the beginning of the 1990s, instituted an institutional framework that is better suited to guaranteeing public liberties in the face of the police powers of the administration, the conciliation between public order and public liberties are still, to the detriment of the latter. This imbalance manifests itself both in the mitigated nature of the legislative framework for judicial review and in the judicial decisions of the judge. On the legislative framework of control, a twofold trend emerges. On the one hand, the often approximative wording of the laws leaves little legibility about the court competent to hear police acts in certain specific matters, which leads to a conflict of competence which is itself liable to result in a denial of justice. On the other hand, there is a gradual liberalization of the contentious procedure relating to public order measures, which is characterized both by the partial lifting of the provisional immunity of police measures, and by the introduction of certain 
procedural garantees to ensure effectiveness of judicial review. As for the judicial policy of the judge, they show the reluctance of the judge to carry out an extensive control of the police measures. This reality results in insufficient control of the facts justifying the police measures, whether the control of their legal characterization or that of their materiality, on the one hand, and the refusal to control the content of the measures, that is, the control of their appropriateness and that of their proportionality, on the other.

\section{INDEX}

Mots-clés : ordre public, liberté publique, contrôle, conciliation, pouvoir

Keywords : public order, public liberty, review, public power, conciliation

\section{AUTEUR}

\section{ÉRIC-ADOL T. GATSI}

Docteur en droit public de l'université de Dschang (Cameroun) Chargé de cours à l'université de Buea (Cameroun) 